\title{
Angiotensin receptor-neprilysin inhibitor in patients with heart failure and chronic kidney disease
}

\author{
In-Jeong Cho ${ }^{1}$, Seok-Min Kang ${ }^{2}$ \\ ${ }^{1}$ Division of Cardiology, Department of Internal Medicine, Ewha Womans University Seoul Hospital, Ewha Womans University College of \\ Medicine, Seoul, Republic of Korea \\ ${ }^{2}$ Division of Cardiology, Department of Internal Medicine, Severance Cardiovascular Hospital, Yonsei University College of Medicine, Seoul, \\ Republic of Korea
}

Despite significant advances in the management of heart failure with reduced ejection fraction (HFrEF), there remains an enormous health problem with high morbidity and mortality over the last few decades. The neprilysin inhibitor enhances the activity of natriuretic peptides, producing vasodilation, natriuresis, and diuresis. Angiotensin receptor blockers inhibit the renin-angiotensin-aldosterone system. Sacubitril/valsartan, a first-in-class angiotensin receptor-neprilysin inhibitor (ARNI), has been shown to improve cardiovascular outcomes in HFrEF and delay the progression of chronic kidney disease (CKD) in patients with HFrEF. The PARADIGM-HF study showed a reduction in diuretic need in the ARNI group. While the use of diuretics is effective in volume control in patients with HFrEF, their use has the potential to adversely affect renal function. Therefore, ARNI therapy could benefit patients with heart failure and CKD by reducing cardiovascular morbidity and mortality and possibly retarding the progression of CKD, although more clinical evidence is required in patients with severe CKD and end-stage renal disease.

Keywords: Chronic kidney disease, Heart failure, Neprilysin, Renin-angiotensin-aldosterone system

\section{Introduction}

Heart failure (HF) and chronic kidney disease (CKD) are expected to continue to increase worldwide as the number of elderly people increases [1,2]. The heart and kidneys are closely related and interdependent, which is expressed by the term cardiorenal syndrome [3]. The presence of concomitant HF and CKD accelerates the presentation and progression of the disease. Patients with both HF and CKD are at an increased risk of hospitalization, need for intensive care or renal replacement, and death [4]. A large meta-analysis of patients with HF found that up to 55\% of HF patients had a reduced estimated glomerular filtration rate (eGFR) of $<60 \mathrm{~mL} / \mathrm{min} / 1.73 \mathrm{~m}^{2}$, and there was a stepwise increase in mortality risk with an increase in CKD stages [5].

There are two major risks for patients with CKD; cardiovascular morbidity or mortality and an increased risk of progression to end-stage renal disease (ESRD) requiring

Received: October 29, 2021; Accepted: October 29, 2021

Editor: Tae-Hyun Yoo, Yonsei University, Seoul, Republic of Korea

Correspondence: Seok-Min Kang

Division of Cardiology, Department of Internal Medicine, Severance Cardiovascular Hospital, Yonsei University College of Medicine, 50 Yonsei-ro, Seodaemun-gu, Seoul 03722, Republic of Korea. E-mail: smkang@yuhs.ac

ORCID: https://orcid.org/0000-0001-9856-9227

Copyright (@ 2021 by The Korean Society of Nephrology

(a) This is an Open Access article distributed under the terms of the Creative Commons Attribution Non-Commercial and No Derivatives License (http:// creativecommons.org/licenses/by-nc-nd/4.0/) which permits unrestricted non-commercial use, distribution of the material without any modifications, and reproduction in any medium, provided the original works properly cited. 
dialysis or kidney transplantation $[6,7]$. Therefore, the comprehensive goal for the management of CKD patients is to prevent cardiovascular disease and attenuate progression to ESRD. As CKD progresses, the clinical manifestation of cardiovascular disease changes from atherosclerotic disease to nonatherosclerotic disease $[8,9]$ and the incidence of HF and sudden cardiac death increases. Unfortunately, the treatment of patients with concomitant HF and CKD is challenging as CKD progresses. Patients with HF and CKD may frequently fail to respond to conventional HF therapies and experience an increased risk of toxicity to guideline-directed medical therapy (GDMT) of HF [10].

Previous studies have shown that inhibition of the renin-angiotensin-aldosterone system (RAAS) decreases the risk of cardiovascular events and slows the progression of CKD with proteinuria [11], suggesting both the cardiovascular and renal benefits of RAAS inhibition in CKD patients. Recently, dual inhibition of neprilysin and RAAS has shown superior cardiovascular and renal benefits compared to conventional RAAS inhibitors, including angiotensin-converting enzyme inhibitors (ACEIs) and angiotensin receptor blockers (ARBs) in patients with HF [12,13]. The first-in-class angiotensin receptor-neprilysin inhibitor (ARNI), sacubitril/valsartan, is rapidly replacing RAAS inhibitors as a frontline medical therapy in patients with heart failure with reduced ejection fraction (HFrEF) [14]. This review explores the background of ARNI in HF and offers guidance on how to use ARNI in clinical practice, especially in patients with concomitant HFrEF and CKD.

\section{Classification of heart failure and guideline- directed medical therapy}

HF was categorized according to left ventricular ejection fraction (LVEF) in the 2016 European Society of Cardiology Guidelines for HF as follows: HF with preserved ejection fraction (HFpEF), LVEF $\geq 50 \%$; HFrEF, LVEF < 40\%; and HF with mid-range ejection fraction, LVEF $40 \%$ to $49 \%$ [15]. More recently, a new revised 2021 universal classification of HF has been proposed, including HFrEF, LVEF $\leq 40 \%$; HF with mildly reduced ejection fraction, LVEF $41 \%$ to 49\%; HFpEF, LVEF $\geq 50 \%$; and HF with improved ejection fraction: a baseline LVEF $\leq 40 \%$, a $\geq 10 \%$ increase from baseline LVEF, and a second measurement of LVEF $>40 \%$ [16]. There is no robust evidence that any treatment can modify the natural history of patients with HFpEF, probably due to the heterogeneity of its etiologies [17]. In contrast, there is plenty of evidence for medical therapy for HFrEF, which has shown survival improvement in large randomized controlled clinical trials, including ACEIs, ARBs, beta-blockers, mineralocorticoid antagonists (MRAs), and an ARNI [18].

RAAS inhibition has been the mainstay of treatment strategies for patients with HFrEF $[19,20]$. Randomized controlled trials have proven that the RAAS plays an important role in the pathophysiology of HFrEF. The blocking points in RAAS for each ARNI, ACEI, ARB, and MRA are systemically demonstrated in Fig. 1. The updated guidelines for HF treatment recommend the use of an ARNI, ACEI, or ARB to reduce morbidity and mortality in patients with chronic HFrEF and advise that patients who can tolerate an ACEI or ARB should change to an ARNI to further reduce adverse cardiovascular outcomes [21,22]. Furthermore, the 2021 American College of Cardiology Expert Consensus has suggested that an ARNI is the preferred method for RAAS inhibition over ACEIs or ARBs if there are no compelling contraindications, suggesting a superior role of ARNI in the management of HFrEF in other RAAS inhibitors [14].

\section{Dual angiotensin receptor-neprilysin inhibition}

In patients with HFrEF, RAAS is upregulated, which leads to excessive production of natriuretic peptides. Consequently, natriuretic peptides modulate the response to RAAS by aiding natriuresis and vasodilation [23]. Neprilysin is responsible for the breakdown of vasoactive peptides. Neprilysin inhibition increases endogenous levels of vasoactive peptides, resulting in increased vasodilation, natriuresis, and diuresis, as well as a reduction in cardiac fibrosis and hypertrophy. However, neprilysin inhibition also impairs the degradation of angiotensin II, which induces compensatory upregulation of RAAS and sympathetic nervous activity [24]. Therefore, the best strategy to suppress RAAS would be to inhibit the breakdown of natriuretic peptides and block the RAAS simultaneously [25], which led to the development of ARNI. Neprilysin inhibitors are not combined with ACEI, since a previous study has shown a higher risk of angioedema with the combination of neprilysin inhibitor and ACEI [26]. The first-in-class ARNI, sacubitril/ valsartan, are the only ARNIs approved for clinical use and have shown many benefits in patients with HFrEF. The 


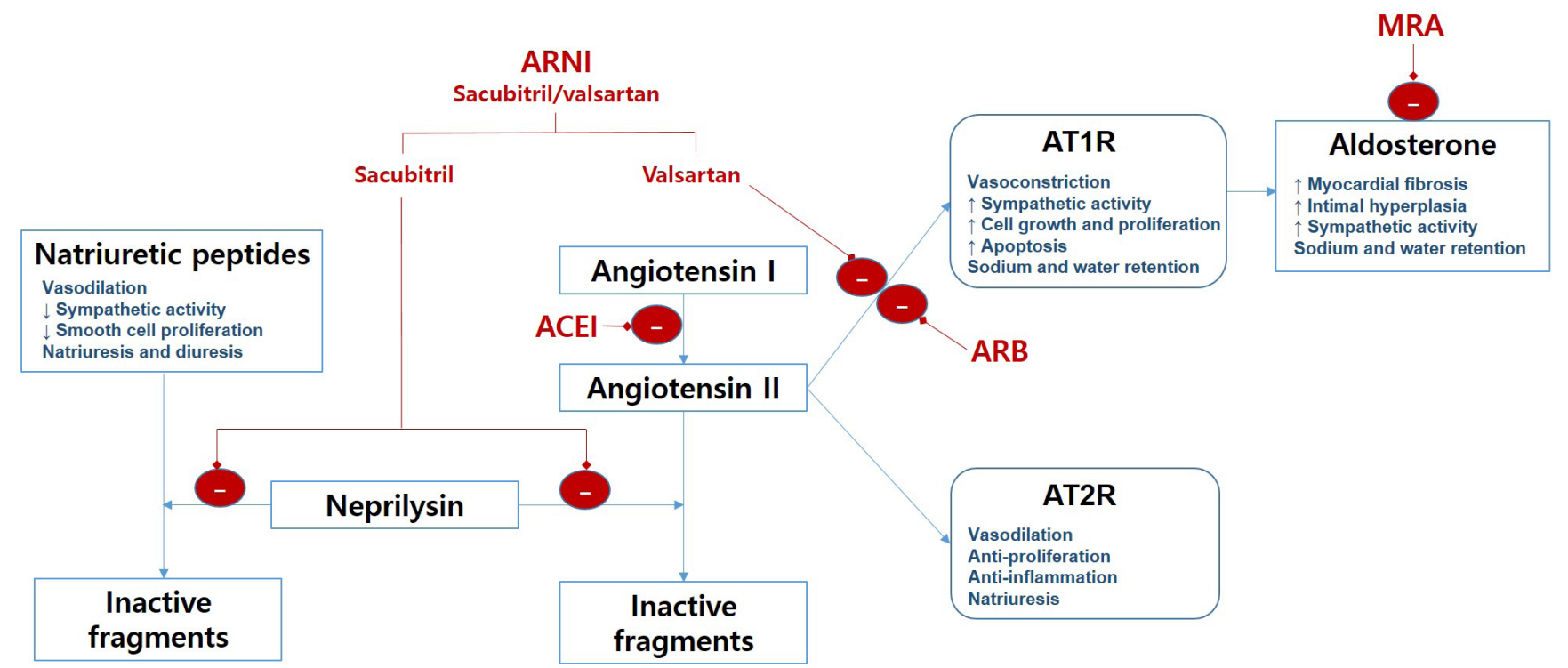

Figure 1. Blocking points for renin-angiotensin-aldosterone system inhibitors and a neprilysin inhibitor.

ACEI, angiotensin-converting enzyme inhibitor; ARB, angiotensin receptor blocker; ARNI, angiotensin receptor-neprilysin inhibitor; AT1R, angiotensin II receptor type 1; AT2R, angiotensin II receptor type 2; MRA, mineralocorticoid antagonist.

indications, contraindications, and cautions for sacubitril/ valsartan use are summarized in Table 1.

\section{Cardiovascular effects of angiotensin receptor- neprilysin inhibitor}

The long-term benefits of sacubitril/valsartan on cardiovascular morbidity and mortality over other RAAS inhibitors in patients with chronic HFrEF was first described in the Prospective Comparison of ARNI with ACEI to Determine Impact on Global Mortality and Morbidity in Heart Failure (PARADIGM-HF) trial, which showed that sacubitril/valsartan was superior to enalapril in reducing the risk of HF hospitalization and cardiovascular death by $20 \%$ [12]. According to the result of PARADIGM-HF, guidelines have recommended sacubitril/valsartan as a replacement for ACEIs or ARBs $[15,18]$. Claggett et al. [27] suggested that the life expectancy of patients receiving ARNI might increase by 1 to 2 years compared with patients receiving ACEI, supporting a strong recommendation to use sacubitril/valsartan for patients with HFrEF. Furthermore, the Prospective Study of Biomarkers, Symptom Improvement, and Ventricular Remodeling During Entresto Therapy for Heart Failure (PROVE-HF) trial [28], Comparison of Sacubitril-Valsartan versus Enalapril on Effect on NT-proBNP in Patients Stabi- lized from an Acute Heart Failure Episode (PIONEER-HF) trial [29], and the Comparison of Pre- and Post-discharge Initiation of LCZ696 Therapy in HFrEF Patients After an Acute Decompensation Event (TRANSITION) study [30] have shown that sacubitril/valsartan was effective and safe in a wide range of HFrEF, including those with acute decompensated HF, newly diagnosed HF, and HF without prior ACEI or ARB use, all of which supports the expansion of ARNI application in a broad range of patients with HFrEF.

In contrast to the promising results from patients with HFrEF, the Prospective Comparison of ARNI with ARB Global Outcomes in HF with Preserved Ejection Fraction (PARAGON-HF) trial in patients with HFpEF showed that sacubitril/valsartan did not result in a significantly lower rate of total HF hospitalizations and cardiovascular deaths among patients with HFpEF (LVEF > 45\%), even though there was a suggestion of possible benefit with sacubitril/ valsartan and in women and in patients with lower LVEF (ejection fraction < 57\%) [31]. The Angiotensin Receptor Neprilysin Inhibition Versus Individualized RAAS blockade (PARALLAX) trial which randomized 2,572 patients with an HFpEF (LVEF > 40\%) showed mixed results, in which only one of two co-primary endpoints showed significant improvement in the sacubitril/valsartan group compared to the comparator (enalapril, valsartan, or placebo), and the 
Table 1. Indications, contraindications, and cautions for the administration of sacubitril/valsartan

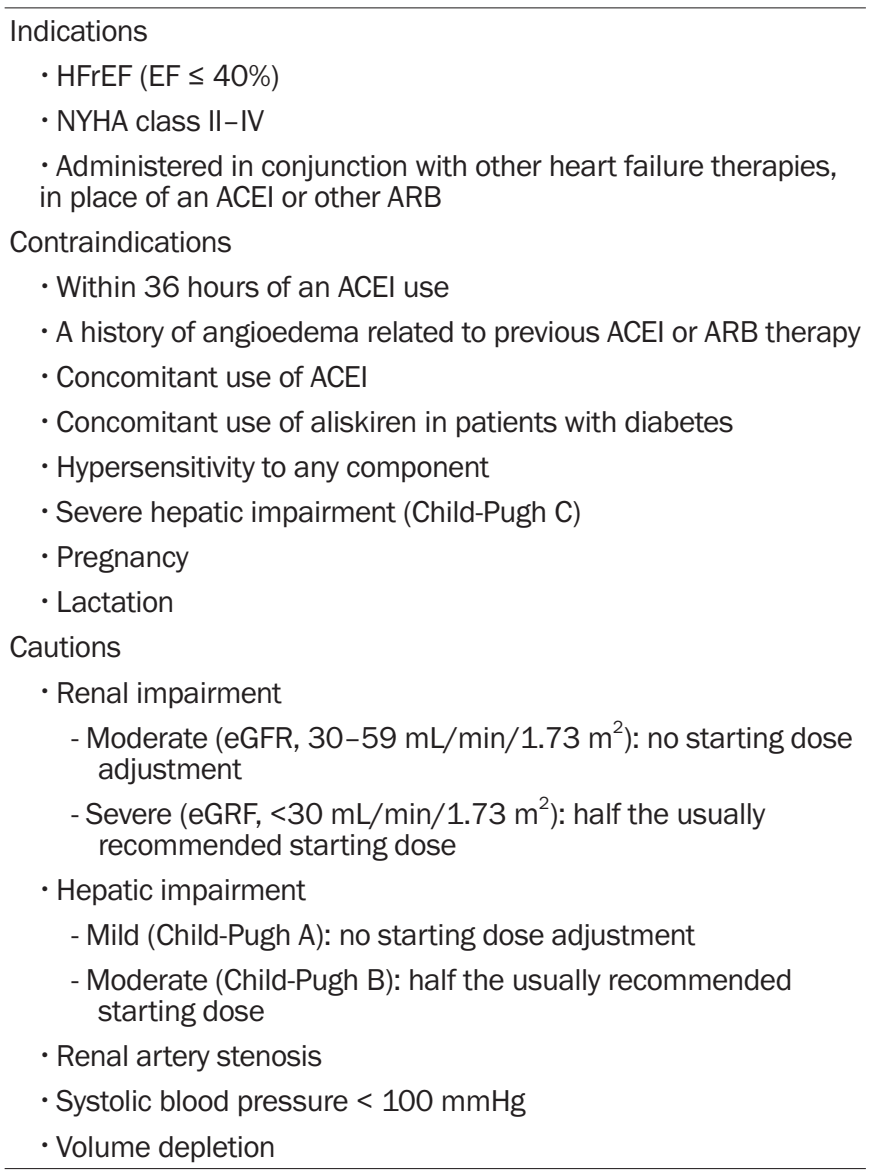

The indications, contraindications, and cautions for sacubitril/valsartan follow the U.S. Food and Drug Administration-approved labeling indications. ACEI, angiotensin-converting enzyme inhibitor; ARB, angiotensin receptor blocker; eGFR, estimated glomerular filtration rate; HFrEF, heart failure with reduced ejection fraction; NYHA, New York Heart Association.

reduction in $\mathrm{N}$-terminal prohormone of brain natriuretic peptide (NT-proBNP) was $16 \%$ greater in the sacubitril/ valsartan group (adjusted geometric mean ratio, 0.84; $95 \%$ confidence interval [CI], 0.80-0.88), while there was no significant difference between groups in the 6-minute walk distance [32]. However, severe adverse events were lower in the sacubitril/valsartan group than in the individualized medical therapy group; first hospitalization due to HF (hazard ratio [HR], 0.49; 95\% CI, 0.30-0.81; $\mathrm{p}=0.005$ ) and composite of death due to HF or HF hospitalization (HR, 0.64; 95\% CI, 0.42-0.97; $\mathrm{p}=0.034$ ) were lower, although they were not the primary endpoints of the PARALLAX trial [32]. The U.S. Food and Drug Administration has recently approved the indication of sacubitril/valsartan in patients with HFpEF with LVEF below normal to reduce worsening HF (total HF hospitalizations and urgent HF visits), although further clarification is still needed for HFpEF subgroups who can benefit mostly. Randomized clinical trials assessing the clinical outcomes of sacubitril/valsartan are summarized in Table 2.

\section{Renal effects of angiotensin receptor-neprilysin inhibitor}

Inhibition of RAAS reduces urinary albumin excretion and delays the progression of CKD to ESRD. However, treatment with RAAS inhibitors is limited in patients with CKD, as the risk of serum creatinine increase or hyperkalemia is greater in CKD patients than in those without this medical condition [11]. RAAS inhibition by ACEIs or ARBs decreases intra-glomerular pressure by preventing angiotensin II-induced predominant vasoconstriction of the efferent arteriole, contributing to a decrease in albuminuria and eGFR [33].

Three natriuretic peptides are present in humans; atrial natriuretic peptide (ANP), brain natriuretic peptide (BNP), and C-type natriuretic peptide [23]. ANP and BNP are synthesized in cardiac myocytes, whereas C-type natriuretic peptide is mainly expressed in endothelial cells [23]. ANP increases renal perfusion through systemic vasodilation, and there is evidence that sacubitril mainly acts by enhancing ANP instead of BNP [34]. Concomitant inhibition of angiotensin II and neprilysin induces selective vasorelaxation of preglomerular afferent arterioles and relative vasoconstriction of the postglomerular efferent arteriole, contributing to increased intracapillary hydraulic pressure and eGFR [35]. Sacubitril/ valsartan may also affect renal tubular reabsorption. By increasing ANP, it inhibits sodium reabsorption in the renal proximal tubule, which may account for the benefits of ARNI therapy in patients with HF [35]. Sacubitril/valsartan has been shown to prevent fibrosis, mitochondrial damage, oxidative stress, and apoptosis in kidney and heart tissues of cardiorenal syndrome rat models [36]. The urine albumin creatinine ratio (ACR) modestly increases after ARNI initiation [37-39], increasing concerns regarding deterioration of kidney function after ARNI use. However, in contrast to worse renal outcome related to the increase in albuminuria with enalapril therapy, an increase in the ACR was not related to worse renal 


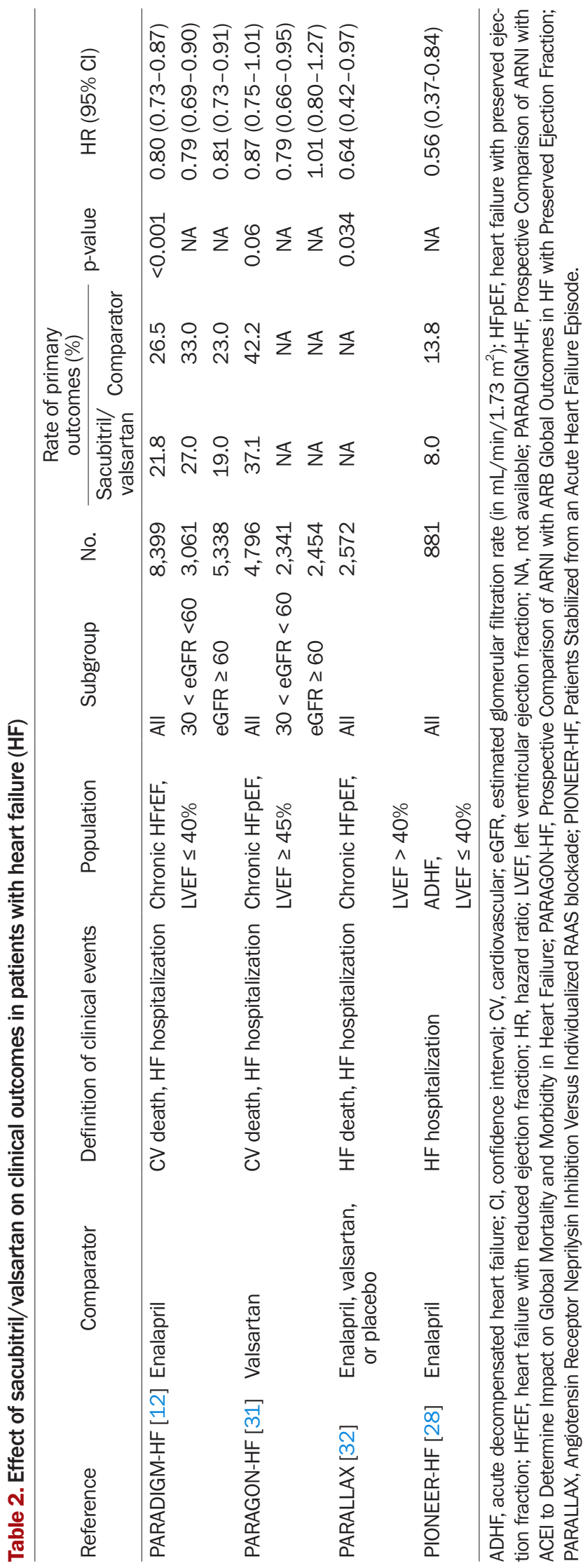

outcome with ARNI therapy, suggesting the increase in the ACR is mediated by a mechanism that does not result in low renal filtration [37]. Despite a similar increase in ACR, the Prospective Comparison of ARNI with ARB on Management of Heart Failure with Preserved Ejection Fraction (PARAMOUNT) trial reported a slower deterioration of eGFR in patients with HFpEF after scubitril/valsartan use. A plausible explanation for this specific dissociation phenomenon between albuminuria and renal function deterioration is the selective vasorelaxation of preglomerular afferent arterioles with ARNI use, leading to an increase in intracapillary hydraulic pressure, which may contribute to increased albumin ultrafiltration and a modest increase in albuminuria without renal function deterioration [35].

The renal safety of sacubitril/valsartan has been reported consistently in patients with HFrEF $[12,37]$ and HFpEF, which included a significant number of patients with stage 2 and 3 CKD (eGFR, 30-59 $\mathrm{mL} / \mathrm{min} / 1.73 \mathrm{~m}^{2}$ ). PARADIGM-HF post-hoc analysis [37] and PARAGON-HF [38] showed that sacubitril/valsartan led to a slower rate of decrease in eGFR and improved renal outcomes in patients with HFrEF and HFpEF. In a study of patients with acute decompensated HF, sacubitril/valsartan showed similar renal event rates to those of enalapril [29]. In a meta-analysis, Kang et al. [40] reported that compared to other RAAS inhibitors, sacubitril/valsartan significantly increased the eGFR and decreased blood pressure, suggesting that it may have renal and cardiovascular benefits in patients with HF and CKD. The efficacy and safety of sacubitril/valsar$\tan$ have also been studied in patients with other cardiovascular or renal diseases, although many recent studies have investigated patients with HF. Sacubitril/valsartan demonstrated a low prevalence of renal side effects including hyperkalemia, hypokalemia, and creatinine elevation in patients with hypertension despite its superior blood pressure-lowering effect compared to olmesartan [41,42]. The United Kingdom Heart and Renal Protection-III (UK HARP-III) trial investigating 414 patients with CKD (eGFR, 20-60 $\mathrm{mL} / \mathrm{min} / 1.73 \mathrm{~m}^{2}$ ) without HF showed that sacubitril/valsartan had similar effects on kidney function and albuminuria to irbesartan, but it has the additional effect of lowering blood pressure and cardiac biomarkers [43]. Randomized clinical trials assessing the renal outcomes of sacubitril/valsartan are summarized in Table 3.

Hyperkalemia is a potentially serious complication in 


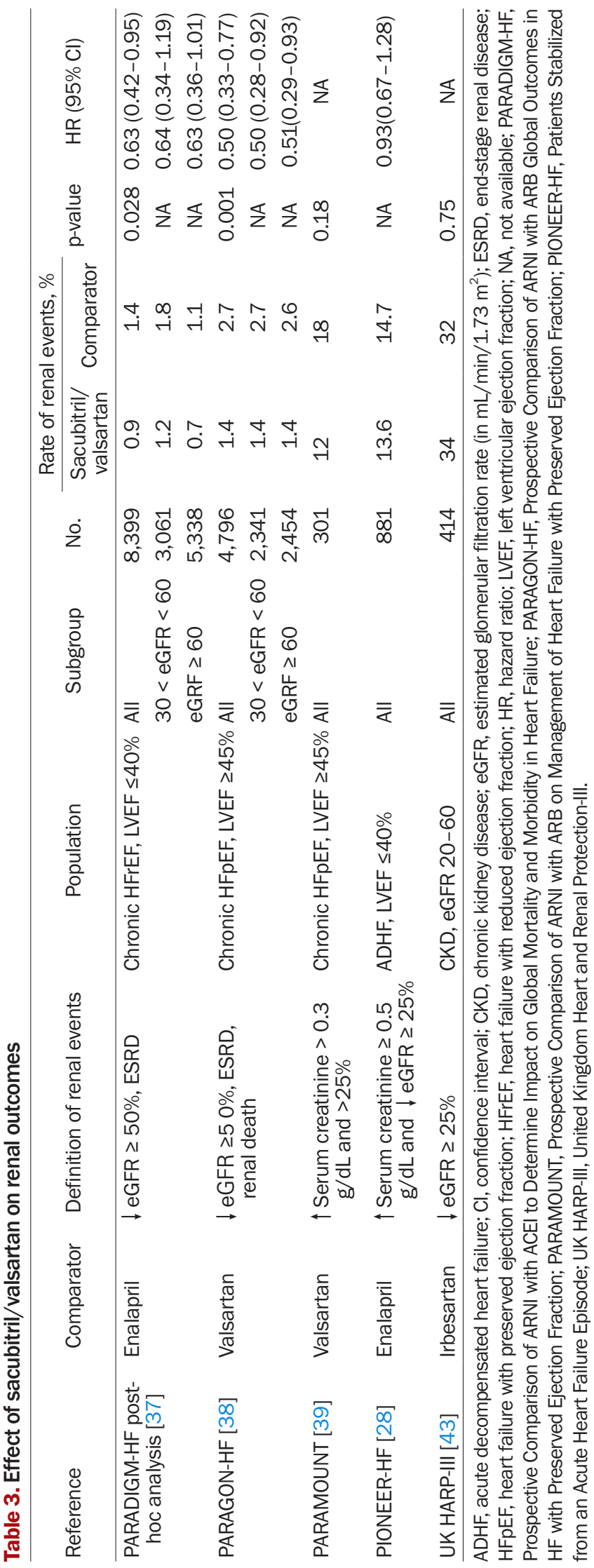

CKD patients receiving RAAS inhibitors, which can impact clinical outcomes directly and can limit the use of GDMT [44]. The benefits of MRA in patients with HFrEF are well established $[45,46]$. However, physicians are reluctant to initiate MRA in patients with CKD due to concerns of hyperkalemia, even though it is recommended to initiate MRA in conjunction with ACEIs, ARBs, or an ARNI to reduce morbidity and mortality in patients with New York Heart Association classes II-IV symptoms [18]. In the PARADIGM-HF trial, potassium levels of $>6.0 \mathrm{mmol} / \mathrm{L}$ occurred in $4 \%$ of the patients treated with sacubitril/valsartan and in $6 \%$ of the patients with enalapril, and the difference was statistically significant [12]. Moreover, sacubitril/valsartan has been reported to attenuate the risk of hyperkalemia when MRAs are combined with other inhibitors of the RAAS system, suggesting the safer use of MRAs when combined with ARNI [47].

\section{Efficacy and safety of angiotensin receptor- neprilysin inhibitor in advanced chronic kidney disease}

After oral administration, sacubitril/valsartan was divided into valsartan and prodrug sacubitril. Valsartan is primarily excreted via the biliary route, and renal impairment does not affect its pharmacokinetics [48]. Sacubitril is rapidly converted to the active neprilysin inhibitor sacubitrilat [49]. Kidney function has an insignificant impact on the disposition of sacubitril, which is excreted through the urine and feces in less than $2 \%$ of the total administered dose [49], whereas sacubitrilat is eliminated primarily via the kidney, suggesting that its exposure is increased with renal function decline [50].

The optimal treatment of HF in patients with stage 4 or 5 CKD (eGFR, $<30 \mathrm{~mL} / \mathrm{min} / 1.73 \mathrm{~m}^{2}$ ) is unclear as there is little evidence regarding this. The area under the concentration-time curve increased by 2.7 -fold in patients with eGFR of $<30 \mathrm{~mL} / \mathrm{min} / 1.73 \mathrm{~m}^{2}$, which raises concerns about the safety and toxicity of sacubitril/valsartan in patients with stage 4 or 5 CKD [50]. Unfortunately, most of the previous randomized clinical trials that guided the management of HFrEF with an ARNI defined CKD as baseline eGFR of $<60$ $\mathrm{mL} / \mathrm{min} / 1.73 \mathrm{~m}^{2}$ and excluded patients with severe CKD (eGFR, $<30 \mathrm{~mL} / \mathrm{min} / 1.73 \mathrm{~m}^{2}$ ) [12,30,39,51-53].

There have been a few studies published on the use of 
sacubitril/valsartan in patients with stage 4 or $5 \mathrm{CKD}$, or ESRD. In a real-world study, Chang et al. [54] showed that patients with stage 4 or 5 CKD treated with sacubitril/valsartan had 28\% fewer cardiovascular deaths or HF hospitalizations than those treated with standard HF treatment, including 102 patients with eGFR of $<30 \mathrm{~mL} / \mathrm{min} / 1.73 \mathrm{~m}^{2}$ among the whole study population of 932 patients with HFrEF [54]. Quiroga et al. [55] investigated 66 patients with stage 1 to 4 CKD and HFrEF (17\% of stage 4 CKD) and found that sacubitril/valsartan was safe in patients with CKD, suggesting stability in CKD progression after 6 months.

There is a paucity of data on the evidence of ARNI in patients with ESRD on maintenance dialysis. Heyse et al. [56] presented a case report of a 67-year-old man with HFrEF due to ischemic cardiomyopathy and renal insufficiency undergoing hemodialysis, who tolerated a moderate dose of 49/51 mg twice daily, and finally showed symptomatic improvement with a reduction in HF biomarkers and left ventricular filling pressure. Only one study evaluated the use of sacubitril/valsartan in patients with HFrEF and ESRD, which showed that sacubitril/valsartan reduced cardiac biomarkers and improved LVEF; the most common adverse event was hypotension, which was corrected with down-titration of the drug dosage [57].

\section{Clinical application of angiotensin receptor- neprilysin inhibitor}

Patients with CKD tend to receive GDMT inappropriately, probably due to concerns about hypotension, renal function deterioration, and hyperkalemia [58]. Patients with CKD were at a higher risk for noncompliance during the run-in period of the PARADIGM-HF trial, supporting the need for closer monitoring during the up-titration of sacubitril/valsartan or conversion to sacubitril/valsartan in CKD patients [59]. In patients with moderate CKD (eGFR, $30-59 \mathrm{~mL} / \mathrm{min} / 1.73 \mathrm{~m}^{2}$ ), no dose adjustment is required at the start of sacubitril/valsartan. However, the starting dose of sacubitril/valsartan should be reduced in patients with severe CKD (eGFR, $<30 \mathrm{~mL} / \mathrm{min} / 1.73 \mathrm{~m}^{2}$ ). The PARADIGM-HF study showed a reduction in diuretic need in the ARNI group, suggesting that treatment with ARNI may reduce the requirement for loop diuretics doses compared to other RAAS inhibitors [60]. Failure to down-titrate the diuretic doses in patients taking sacubitril/valsartan in response to reduced clinical need, which may result in over-diuresis that can contribute to hypotension or renal function deterioration [60]. This possibility highlights the significance of assessment and adjustment of diuretic doses prior to and following the initiation of an ARNI. Renal function and potassium levels are recommended to be evaluated within 1 to 2 weeks after ARNI initiation or dose escalation, and the schedule for subsequent monitoring should be determined by the patient's kidney function and volume status [14]. The recommended following intervals for renal function monitoring are monthly for the first 3 months and every 3 months thereafter [14].

\section{Gaps in the evidence and future directions}

The burden of HF in patients with CKD is considerable. However, many clinical trials in HF patients have excluded patients with severe CKD or ESRD, which results in uncertain efficacy and safety of the treatments in the advanced CKD population. ARNI seems to be a promising treatment option that could reduce the risk of cardiovascular morbidity and mortality in patients with CKD, but randomized clinical trials with ARNI have also excluded patients with advanced CKD. Future trials of HF interventions should focus on pre-specified subgroups with eGFR of $<30 \mathrm{~mL} /$ $\min / 1.73 \mathrm{~m}^{2}$.

Newer treatments for HF, such as sodium-glucose cotransporter 2 (SGLT2) inhibitors, are being tested in large clinical trials in both HF and CKD populations [61-64]. Among patients with CKD, the risk of a composite endpoint of a decline in the eGFR of more than $50 \%$, ESRD, or renal or cardiovascular deaths were reduced by $39 \%$ with dapagliflozin than with placebo [61]. However, the benefits of SGLT2 inhibitors for HFrEF management in patients with severe CKD remain unclear. Currently, the use of dapagliflozin and empagliflozin is recommended in patients with eGFR of $\geq 30 \mathrm{~mL} / \mathrm{min} / 1.73 \mathrm{~m}^{2}$ and $\geq 20 \mathrm{~mL} / \mathrm{min} / 1.73$ $\mathrm{m}^{2}$, respectively, since the glucosuric effects of SGLT2 inhibitors may be reduced in those with a lower eGFR. There are little data assessing the combination of an ARNI and an SGLT2 inhibitor, even though the benefit of SGLT2 inhibition was consistent in patients already treated with an ARNI in both Dapagliflozin And Prevention Of Adverse Outcomes In Heart Failure (DAPA-HF) and Empagliflozin 
Outcome Trial in Patients With Chronic Heart Failure with Reduced Ejection Fraction (EMPEROR-Reduced) [65,66]. Further evidence to guide the concomitant use of ARNI and SGLT2 inhibitors is needed. Sotagliflozin, a dual sodium-glucose cotransporter 1 (SGLT1) and SGLT2 inhibitor, resulted in significantly lower cardiovascular death and hospitalizations and urgent visits for HF than placebo in patients with diabetes and recent worsening HF [67]. However, further studies are needed on the concomitant use of SGLT1/SGLT2 inhibitors and ARNIs.

\section{Conclusions}

The heart and kidneys were highly interdependent. CKD is associated with two major risks: fatal or nonfatal cardiovascular diseases and an increased risk of progression to ESRD requiring treatment with renal replacement therapy. ARNI therapy could benefit patients with HF and CKD by reducing cardiovascular morbidity and mortality and possibly retarding the progression of CKD, although more clinical evidence is required in patients with severe CKD and ESRD.

\section{Conflicts of interest}

All authors have no conflicts of interest to declare.

\section{Authors' contributions}

Conceptualization, Investigation, Project administration: IJC, SMK

Formal analysis: IJC

Writing-original draft: IJC

Writing-review \& editing: SMK

All authors read and approved the final manuscript.

\section{ORCID}

In-Jeong Cho, https://orcid.org/0000-0002-1209-5129

Seok-Min Kang, https://orcid.org/0000-0001-9856-9227

\section{References}

1. Lee JH, Kim MS, Kim EJ, et al. KSHF Guidelines for the Management of Acute Heart Failure: part I. Definition, epidemiology and diagnosis of acute heart failure. Korean Circ J 2019;49:1-21.

2. GBD Chronic Kidney Disease Collaboration. Global, regional, and national burden of chronic kidney disease, 1990-2017: a systematic analysis for the Global Burden of Disease Study 2017. Lancet 2020;395:709-733.

3. Rangaswami J, Bhalla V, Blair J, et al. Cardiorenal syndrome: classification, pathophysiology, diagnosis, and treatment strategies: a scientific statement from the American Heart Association. Circulation 2019;139:e840-e878.

4. House AA, Wanner C, Sarnak MJ, et al. Heart failure in chronic kidney disease: conclusions from a Kidney Disease: Improving Global Outcomes (KDIGO) Controversies Conference. Kidney Int 2019;95:1304-1317.

5. McAlister FA, Ezekowitz J, Tarantini L, et al. Renal dysfunction in patients with heart failure with preserved versus reduced ejection fraction: impact of the new Chronic Kidney Disease-Epidemiology Collaboration Group formula. Circ Heart Fail 2012;5:309-314.

6. Manjunath G, Tighiouart H, Ibrahim H, et al. Level of kidney function as a risk factor for atherosclerotic cardiovascular outcomes in the community. J Am Coll Cardiol 2003;41:47-55.

7. Gansevoort RT, Correa-Rotter R, Hemmelgarn BR, et al. Chronic kidney disease and cardiovascular risk: epidemiology, mechanisms, and prevention. Lancet 2013;382:339-352.

8. Foley RN, Parfrey PS, Harnett JD, Kent GM, Murray DC, Barre PE. Impact of hypertension on cardiomyopathy, morbidity and mortality in end-stage renal disease. Kidney Int 1996;49:13791385.

9. Judge PK, Haynes R. TaleNeprilysin and neprilysin inhibition in chronic kidney disease. Curr Opin Nephrol Hypertens 2021;30:123-130.

10. House AA. Cardiorenal syndrome: new developments in the understanding and pharmacologic management. Clin J Am Soc Nephrol 2013;8:1808-1815.

11. Clark H, Krum H, Hopper I. Worsening renal function during renin-angiotensin-aldosterone system inhibitor initiation and long-term outcomes in patients with left ventricular systolic dysfunction. Eur J Heart Fail 2014; 16:41-48.

12. McMurray JJ, Packer M, Desai AS, et al. Angiotensin-neprilysin inhibition versus enalapril in heart failure. $N$ Engl J Med 2014;371:993-1004.

13. Dewan P, Docherty KF, McMurray JJ. Sacubitril/valsartan in Asian patients with heart failure with reduced ejection fraction. Korean Circ J 2019;49:469-484.

14. Writing Committee, Maddox TM, Januzzi JL Jr, et al. 2021 Update 
to the 2017 ACC Expert Consensus Decision Pathway for Optimization of Heart Failure Treatment: answers to 10 pivotal issues about heart failure with reduced ejection fraction. A report of the American College of Cardiology Solution Set Oversight Committee. J Am Coll Cardiol 2021;77:772-810.

15. Ponikowski P, Voors AA, Anker SD, et al. 2016 ESC Guidelines for the diagnosis and treatment of acute and chronic heart failure: the Task Force for the diagnosis and treatment of acute and chronic heart failure of the European Society of Cardiology (ESC). Developed with the special contribution of the Heart Failure Association (HFA) of the ESC. Eur Heart J 2016;37:21292200.

16. Bozkurt B, Coats A, Tsutsui H, et al. Universal definition and classification of heart failure: a report of the Heart Failure Society of America, Heart Failure Association of the European Society of Cardiology, Japanese Heart Failure Society and Writing Committee of the Universal Definition of Heart Failure. Endorsed by the Canadian Heart Failure Society, Heart Failure Association of India, Cardiac Society of Australia and New Zealand, and Chinese Heart Failure Association. Eur J Heart Fail 2021;23:352-380.

17. Cleland JG, Pellicori P, Dierckx R. Clinical trials in patients with heart failure and preserved left ventricular ejection fraction. Heart Fail Clin 2014;10:511-523.

18. Yancy CW, Jessup M, Bozkurt B, et al. 2017 ACC/AHA/HFSA focused update of the 2013 ACCF/AHA Guideline for the management of heart failure: a report of the American College of Cardiology/American Heart Association Task Force on Clinical Practice Guidelines and the Heart Failure Society of America. Circulation 2017;136:e137-e161.

19. Brenner BM, Cooper ME, de Zeeuw D, et al. Effects of losartan on renal and cardiovascular outcomes in patients with type 2 diabetes and nephropathy. N Engl J Med 2001;345:861-869.

20. Jackson CE, Solomon SD, Gerstein HC, et al. Albuminuria in chronic heart failure: prevalence and prognostic importance. Lancet 2009;374:543-550.

21. Writing Committee members; Yancy CW, Jessup M, et al. 2016 ACC/AHA/HFSA focused update on new pharmacological therapy for heart failure. An update of the 2013 ACCF/AHA Guideline for the management of heart failure: a report of the American College of Cardiology/American Heart Association Task Force on Clinical Practice Guidelines and the Heart Failure Society of America. Circulation 2016;134:e282-e293.

22. Ponikowski P, Voors AA, Anker SD, et al. 2016 ESC Guidelines for the diagnosis and treatment of acute and chronic heart failure: the Task Force for the diagnosis and treatment of acute and chronic heart failure of the European Society of Cardiology (ESC). Developed with the special contribution of the Heart Failure Association (HFA) of the ESC. Eur J Heart Fail 2016;18:891-975.

23. Daniels LB, Maisel AS. Natriuretic peptides. J Am Coll Cardiol 2007;50:2357-2368.

24. Corti R, Burnett JC Jr, Rouleau JL, Ruschitzka F, Lüscher TF. Vasopeptidase inhibitors: a new therapeutic concept in cardiovascular disease? Circulation 2001;104:1856-1862.

25. Jhund PS, McMurray JJ. The neprilysin pathway in heart failure: a review and guide on the use of sacubitril/valsartan. Heart 2016; 102:1342-1347.

26. Packer M, Califf RM, Konstam MA, et al. Comparison of omapatrilat and enalapril in patients with chronic heart failure: the Omapatrilat Versus Enalapril Randomized Trial of Utility in Reducing Events (OVERTURE). Circulation 2002;106:920-926.

27. Claggett B, Packer M, McMurray JJ, et al. Estimating the longterm treatment benefits of sacubitril-valsartan. $N$ Engl J Med 2015;373:2289-2290.

28. Velazquez EJ, Morrow DA, DeVore AD, et al. Angiotensin-neprilysin inhibition in acute decompensated heart failure. $N$ Engl $J$ Med 2019;380:539-548.

29. Ambrosy AP, Braunwald E, Morrow DA, et al. Angiotensin receptor-neprilysin inhibition based on history of heart failure and use of renin-angiotensin system antagonists. J Am Coll Cardiol 2020;76:1034-1048.

30. Wachter R, Senni M, Belohlavek J, et al. Initiation of sacubitril/ valsartan in haemodynamically stabilized heart failure patients in hospital or early after discharge: primary results of the randomised TRANSITION study. Eur J Heart Fail 2019;21:998-1007.

31. Solomon SD, McMurray JJV, Anand IS, et al. Angiotensin-neprilysin inhibition in heart failure with preserved ejection fraction. N Engl J Med 2019;381:1609-1620.

32. Pieske B, Shah S, Wachter R, et al. Angiotensin receptor neprilysin inhibition compared with individualized medical therapy for comorbidities in patients with heart failure and preserved ejection fraction: the PARALLAX trial. Paper presented at: 2020 European Society of Cardiology Congress; 2020 Aug 29-Sep 2; Amsterdam, Netherlands.

33. Kobori H, Mori H, Masaki T, Nishiyama A. Angiotensin II blockade and renal protection. Curr Pharm Des 2013;19:3033-3042.

34. Ibrahim NE, McCarthy CP, Shrestha S, et al. Effect of neprilysin inhibition on various natriuretic peptide assays. J Am Coll Cardiol 2019;73:1273-1284.

35. Tersalvi G, Dauw J, Martens P, Mullens W. Impact of sacubi- 
tril-valsartan on markers of glomerular function. Curr Heart Fail Rep 2020;17:145-152.

36. Yang CC, Chen YT, Chen $\mathrm{CH}$, et al. The therapeutic impact of entresto on protecting against cardiorenal syndrome-associated renal damage in rats on high protein diet. Biomed Pharmacother 2019;116:108954.

37. Damman K, Gori M, Claggett B, et al. Renal effects and associated outcomes during angiotensin-neprilysin inhibition in heart failure. JACC Heart Fail 2018;6:489-498.

38. Mc Causland FR, Lefkowitz MP, Claggett B, et al. Angiotensin-neprilysin inhibition and renal outcomes in heart failure with preserved ejection fraction. Circulation 2020;142:1236-1245.

39. Voors AA, Gori M, Liu LC, et al. Renal effects of the angiotensin receptor neprilysin inhibitor LCZ696 in patients with heart failure and preserved ejection fraction. Eur J Heart Fail 2015; 17:510-517.

40. Kang H, Zhang J, Zhang X, et al. Effects of sacubitril/valsartan in patients with heart failure and chronic kidney disease: a meta-analysis. Eur J Pharmacol 2020;884:173444.

41. Cheung DG, Aizenberg D, Gorbunov V, Hafeez K, Chen CW, Zhang J. Efficacy and safety of sacubitril/valsartan in patients with essential hypertension uncontrolled by olmesartan: a randomized, double-blind, 8-week study. J Clin Hypertens (Greenwich) 2018;20:150-158.

42. Supasyndh O, Wang J, Hafeez K, Zhang Y, Zhang J, Rakugi H. Efficacy and safety of sacubitril/valsartan (LCZ696) compared with olmesartan in elderly Asian patients ( $\geq 65$ years) with systolic hypertension. Am J Hypertens 2017;30:1163-1169.

43. Haynes R, Judge PK, Staplin N, et al. Effects of sacubitril/valsartan versus irbesartan in patients with chronic kidney disease. Circulation 2018;138:1505-1514.

44. Ferreira JP, Butler J, Rossignol P, et al. Abnormalities of potassium in heart failure: JACC state-of-the-art review. J Am Coll Cardiol 2020;75:2836-2850.

45. Pitt B, Zannad F, Remme WJ, et al. The effect of spironolactone on morbidity and mortality in patients with severe heart failure. Randomized Aldactone Evaluation Study Investigators. $N$ Engl J Med 1999;341:709-717.

46. Pitt B, Remme W, Zannad F, et al. Eplerenone, a selective aldosterone blocker, in patients with left ventricular dysfunction after myocardial infarction. N Engl J Med 2003;348:1309-1321.

47. Desai AS, Vardeny O, Claggett B, et al. Reduced risk of hyperkalemia during treatment of heart failure with mineralocorticoid receptor antagonists by use of sacubitril/valsartan compared with enalapril: a secondary analysis of the PARADIGM-HF trial.
JAMA Cardiol 2017;2:79-85.

48. Brookman LJ, Rolan PE, Benjamin IS, et al. Pharmacokinetics of valsartan in patients with liver disease. Clin Pharmacol Ther 1997;62:272-278.

49. Flarakos J, Du Y, Bedman T, et al. Disposition and metabolism of [(14)C] sacubitril/valsartan (formerly LCZ696) an angiotensin receptor neprilysin inhibitor, in healthy subjects. Xenobiotica 2016;46:986-1000.

50. Ayalasomayajula SP, Langenickel TH, Jordaan P, et al. Effect of renal function on the pharmacokinetics of LCZ696 (sacubitril/ valsartan), an angiotensin receptor neprilysin inhibitor. Eur J Clin Pharmacol 2016;72:1065-1073.

51. Senni M, McMurray JJ, Wachter R, et al. Initiating sacubitril/valsartan (LCZ696) in heart failure: results of TITRATION, a double-blind, randomized comparison of two uptitration regimens. Eur J Heart Fail 2016;18:1193-1202.

52. Velazquez EJ, Morrow DA, DeVore AD, et al. Rationale and design of the comParIson Of sacubitril/valsartaN versus Enalapril on Effect on nt-pRo-bnp in patients stabilized from an acute Heart Failure episode (PIONEER-HF) trial. Am Heart J 2018;198:145-151.

53. Solomon SD, Zile M, Pieske B, et al. The angiotensin receptor neprilysin inhibitor LCZ696 in heart failure with preserved ejection fraction: a phase 2 double-blind randomised controlled trial. Lancet 2012;380:1387-1395.

54. Chang HY, Feng AN, Fong MC, et al. Sacubitril/valsartan in heart failure with reduced ejection fraction patients: Real world experience on advanced chronic kidney disease, hypotension, and dose escalation. J Cardiol 2019;74:372-380.

55. Quiroga B, de Santos A, Sapiencia D, Saharaui Y, Álvarez-Chiva V. Sacubitril/valsartan in chronic kidney disease, the nephrologist point of view. Nefrologia (Engl Ed) 2019;39:646-652.

56. Heyse A, Manhaeghe L, Mahieu E, Vanfraechem C, Van Durme F. Sacubitril/valsartan in heart failure and end-stage renal insufficiency. ESC Heart Fail 2019;6:1331-1333.

57. Lee S, Oh J, Kim H, et al. Sacubitril/valsartan in patients with heart failure with reduced ejection fraction with end-stage of renal disease. ESC Heart Fail 2020;7:1125-1129.

58. Heywood JT, Fonarow GC, Costanzo MR, et al. High prevalence of renal dysfunction and its impact on outcome in 118,465 patients hospitalized with acute decompensated heart failure: a report from the ADHERE database. J Card Fail 2007;13:422-430.

59. Desai AS, Solomon S, Claggett B, et al. Factors associated with noncompletion during the run-in period before randomization and influence on the estimated benefit of LCZ696 in the PARA- 
DIGM-HF trial. Circ Heart Fail 2016;9:e002735.

60. Vardeny O, Claggett B, Kachadourian J, et al. Reduced loop diuretic use in patients taking sacubitril/valsartan compared with enalapril: the PARADIGM-HF trial. Eur J Heart Fail 2019;21:337341.

61. Heerspink HJ, Stefánsson BV, Correa-Rotter R, et al. Dapagliflozin in patients with chronic kidney disease. $N$ Engl J Med 2020;383:1436-1446.

62. Heerspink HJ, Stefansson BV, Chertow GM, et al. Rationale and protocol of the Dapagliflozin And Prevention of Adverse outcomes in Chronic Kidney Disease (DAPA-CKD) randomized controlled trial. Nephrol Dial Transplant 2020;35:274-282.

63. Herrington WG, Preiss D, Haynes R, et al. The potential for improving cardio-renal outcomes by sodium-glucose co-transport- er-2 inhibition in people with chronic kidney disease: a rationale for the EMPA-KIDNEY study. Clin Kidney J2018;11:749-761.

64. Oh J, Lee SH, Lee CJ, Kang SM. Sodium-glucose co-transporter 2 inhibitors: a new path for heart failure treatment. Korean Circ J 2021;51:399-408.

65. McMurray J, Solomon SD, Inzucchi SE, et al. Dapagliflozin in patients with heart failure and reduced ejection fraction. $N$ Engl JMed 2019;381:1995-2008.

66. Packer M, Anker SD, Butler J, Filippatos G, et al. Cardiovascular and renal outcomes with empagliflozin in heart failure. $N$ Engl J Med 2020;383:1413-1424.

67. Bhatt DL, Szarek M, Steg PG, et al. Sotagliflozin in patients with diabetes and recent worsening heart failure. N Engl J Med 2021; 384:117-128. 